\title{
Ocorrência de ectoparasitas no roedor Oligoryzomys microtis em regiões ao norte do Departamento de La Paz, Bolívia
}

[Occurrence of ectoparasites in rodents of the species Oligoryzomys microtis in northern La Paz Department, Bolivia]

\author{
M.S.C. Graça ${ }^{1}$, P.A.S. Basto ${ }^{2}$, A. Rico ${ }^{4,6}$, J. Martinez ${ }^{3}$, I.V. Sanchez-Roman ${ }^{3}$, E.I. Alandia ${ }^{6}$, \\ M.I. Moya ${ }^{5}$, S. Revollo-Cadima ${ }^{6}$, J. Salazar-Bravo ${ }^{5,6,7}$ \\ ${ }^{1}$ Aluna de graduação - Faculdade de Medicina Veterinária - Universidade Metropolitana de Santos - Santos, SP \\ ${ }^{2}$ Mestrado Saúde e Meio Ambiente - Universidade Metropolitana de Santos - Santos, SP \\ ${ }^{3}$ Carrera de Biología, Universidad Mayor de San Andrés - La Paz, Bolívia \\ ${ }^{4}$ Global Change Research Institute Bĕlidla - Brno, Tchéquia \\ ${ }^{5}$ Colección Boliviana de Fauna, Museo Nacional de Historia Natural de Bolivia \\ ${ }^{6}$ Instituto de Ecologia, Carrera de Biología, Facultad de Ciencias Puras y Naturales, Universidad Mayor de San \\ Andrés - La Paz, Bolívia. \\ ${ }^{7}$ Department of Biological Sciences, Texas Tech University - Lubbock, Estados Unidos
}

\section{RESUMO}

O objetivo deste trabalho foi mapear e descrever a ocorrência de roedores e seus ectoparasitas nas regiões norte do departamento de La Paz, Bolívia. De abril a maio e julho a agosto de 2017, 80 indivíduos da espécie Oligoryzomys microtis foram capturados em armadilhas vivas. Uma amostra aleatória de 36 indivíduos foi analisada quanto à presença de ectoparasitas, encontrando um total de 458 ectoparasitas. Os roedores foram capturados em dois tipos de ambientes: floresta secundária e áreas cultivadas, sendo a área cultivada a área com maior captação de roedores. Dos ectoparasitas, Laelaps sp. foi o gênero com maior abundância e Mysolaelaps sp. o mais prevalente; o gênero Polygenis sp. é aparentemente descrito pela primeira vez na selva amazônica, exigindo estudos adicionais para entender melhor os patógenos que são transmitidos no parasitismo desta pulga em roedores devido à sua importância no ecossistema e para a saúde pública.

Palavras-chave: ectoparasitas, Oligoryzomys, roedores

\begin{abstract}
The objective of this work was to map and describe the occurrence of rodents and their ectoparasites in regions to the North of the department of La Paz in Bolivia. From April to May and July to August 2017, 80 Oligoryzomys microtis rodents were captured and 36 random samples of their ectoparasites were analyzed, totaling 458 ectoparasites. Rodents were captured in two types of environments: secondary forest and crops areas, being the second one with greater amount of capture of rodents. Among the ectoparasites, Laelaps sp was the genus with the highest abundance and Mysolaelaps sp with the highest prevalence; the genus Polygenis sp is apparently described for the first time in the Amazon rainforest, requiring more studies to better understand the pathogens transmitted in the parasitism of this flea in rodents due to their importance in the ecosystem and public health.
\end{abstract}

Keywords: ectoparasites, Oligoryzomys, rodents

\section{INTRODUÇÃO}

Os animais silvestres, tanto em vida silvestre como em cativeiro, podem ser reservatórios e portadores de patógenos causadores de zoonoses
(Silva, 2004). Entre os diversos patógenos transmitidos por roedores, podem ser citados o Hantavirus e a Leptospira, por causarem perdas econômicas, além da grande importância para a saúde pública devido à elevada letalidade,

Recebido em 9 de julho de 2018

Aceito em 25 de janeiro de 2019

E-mail: mi.satorelli@gmail.com 
principalmente por Hantavirus (Körting et al., 2008; Castro et al., 2009). Alguns fatores aumentam a ocorrência de zoonoses, principalmente pela predação do meio ambiente pelo homem (Estevam e Job, 2016). Os roedores Oligoryzomys são o gênero mais especioso entre os Oryzomyini, com distribuição desde o norte da América Central até a América do Sul. No Brasil o gênero se distribui na Amazônia, na Caatinga, no Pantanal, no Cerrado, na Mata Atlântica e nos Campos Sulinos (Machado et al., 2011), enquanto na Bolívia o gênero está presente em praticamente todo o país (Anderson, 1997).

Os roedores Sigmodontine são um componente fundamental da fauna e são hospedeiros de inúmeras espécies de ectoparasitas, epidemiologicamente, importantes porque envolvem a transmissão de agentes patogênicos cujos reservatórios são roedores (Oliveira et al., 2014). A maioria desses ácaros que infestam o homem e os animais pertence a três famílias: Laelapidae, que parasitam pequenos mamíferos (roedores e marsupiais), sendo encontrados na pelagem e em ninhos de seus hospedeiros (Reis et al., 2008) - ressalta-se que os representantes da ordem Phthiraptera são ectoparasitas obrigatórios, permanentes, hematófagos e altamente específicos (Lareschi e López, 2000); Ixodidae, que parasitam quase todas as espécies de mamíferos sinantrópicos, silvestres e domésticos, inclusive o homem, as aves, os répteis e os anfíbios. Além da espoliação direta e da inoculação de toxinas, podem ser vetores, transmitindo agentes patogênicos - membros da ordem Siphonaptera parasitam animais de sangue quente. São agentes infestantes atuando como parasitas e como vetores (Reis et al., 2008). Considerando o exposto, o objetivo deste trabalho foi mapear e descrever a ocorrência de roedores e seus ectoparasitas em regiões ao norte do estado de La Paz, na Bolívia.

\section{MATERIAL E METÓDOS}

Entre abril e maio, julho e agosto de 2017, período de outono-inverno, foram coletados 221 roedores de seis regiões: sendo quatro parcelas de amostragem em cada local, para ter amostras de floresta e cultivo: Buena Vista (floresta e cultivo), Tumupasa (floresta e cultivo), Sadiri (floresta), Altamarani (floresta e cultivo), EASBA (Empresa
Açucareira San Buenaventura) (cultivo) e Jacj Cuisi (floresta); todos provenientes do município de San Buenaventura, no departamento de La Paz, Bolívia. Foram colocadas por região 400 armadilhas modelo Sherman, durante cinco dias, em cada uma das seis regiões, totalizando cinco dias de colocação de armadilhas por região. Em cada intervalo de 10 metros, colocavam-se cinco linhas de armadilhas, sendo cinco pontos em cada linha, com duas armadilhas por ponto, tendo uma distância de aproximadamente $1,5 \mathrm{~m}$ de cada ponto, totalizando 50 armadilhas por parcela. As armadilhas foram colocadas em floresta ou cultivo; procurava-se por 200 armadilhas em área de floresta e 200 em área de cultivo, cada lugar era marcado e identificado com um auxílio de uma “flag" biodegradável. Para atrair os roedores, foi utilizada como isca uma mistura de aveia, essência de baunilha, atum enlatado e água.

Os ectoparasitas colhidos foram anestesiados com éter e coletados por remoção mecânica, com auxílio de escova, pinça hemostática, e colocados em microtubos com álcool 70\%, sendo um microtubo por roedor. Os ectoparasitas dos microtubos a serem analisados foram colocados em outro microtubo, em líquido de Hertwigs, e identificados com seu número de dados de controle, com a região e o dia em que foram coletados, a fim de serem transparentados e colocados em banho-maria a $70^{\circ} \mathrm{C}$, com temporizador por 12 horas. Após transparentados, os ectoparasitas foram identificados por gênero, considerando-se as características morfológicas, com o uso de microscópio. Foram selecionados aleatoriamente 36 microtubos, analisando-se 15 amostras por região. Regiões com menos de 15 amostras foram analisadas todas elas (Tab. 1).

Os cálculos foram realizados (Bush et al., 1997) e foi considerada abundância média aquela igual ao número total de uma espécie particular de parasita (ou grupo taxonômico) em uma amostra de uma espécie particular de hospedeiro, dividido pelo número total de hospedeiros da espécie, incluindo infectado e não infectado. A prevalência foi igual ao número de hospedeiros infectados em um ou mais indivíduos de uma espécie particular de parasita (ou grupo taxonômico), dividido pelo número de hospedeiros examinados para uma espécie de parasita, multiplicado por 100. 
Ocorrência de ectoparasitas...

Tabela 1. Quantidade de microtubos coletados e analisados de roedores da espécie Oligoryzomys microtis por região de sua área (floresta ou plantação) correspondente.

\begin{tabular}{lcccc}
\hline Região & \multicolumn{5}{c}{ Quantidade de microtubos } \\
\hline Buena Vista & Coletados & Analisados & Floresta & Plantação \\
\hline Tumupasa & 03 & 03 & 0 & 03 \\
EASBA & 02 & 02 & 0 & 02 \\
Altamarani & 33 & 15 & 02 & 13 \\
Jacj Cuisi & 41 & 15 & 02 & 13 \\
Total & 01 & 01 & 01 & 0 \\
\hline
\end{tabular}

\section{RESULTADOS}

Foram capturados 221 roedores, sendo 80 do gênero Oligoryzomys microtis. Deste, por cálculo de poder de amostra, somente 36 amostras foram analisadas, e formaram-se conjuntos de três microtubos de ectoparasitas que se referiam a Buena Vista, dois a Tumupasa, 15 a Altamarani, 15 a EASBA, um a Jacj Cuisi. A região de Sadiri foi omitida, pois não houve captura de Oligoryzomys microtis. Dos resultados de prevalência de roedores Oligoryzomys microtis, $13,89 \%$ (5/36) correspondem à área de floresta e $86,11 \%$ (31/36) ao cultivo. Houve uma prevalência de $25 \%$ (9/36) dos roedores em idade adulta e $75 \%$ em idade subadulta (27/36).

Os ectoparasitas analisados constituíram os grupos $\mathbf{M}_{1}, \mathbf{M}_{2}, \mathbf{M}_{3}, \mathrm{M}_{5}, \mathrm{M}_{6}, \mathrm{M}_{7}$ e $\mathrm{M}_{8}$, com representantes da ordem Acari, e o grupo $\mathrm{M}_{4}$, com representante da ordem Siphonaptera. O grupo $\mathrm{M}_{1}$ correspondeu à família Laelapidae e ao gênero
Laelaps sp.; o $\mathrm{M}_{2}$, à família Laelapidae e ao gênero Mysolaelaps sp.; o $\mathrm{M}_{3}$, à família Laelapidae e ao gênero Gigantolaelaps sp.; $\mathrm{M}_{4}$, à família Rhopalopsylidae e ao gênero Polygenis sp.; o $\mathrm{M}_{5}$, à família Laelapidae e ao gênero Androlaelaps sp. 1; o $\mathrm{M}_{6}$, à família Laelapidae o gênero não pôde ser identificado por não apresentar íntegras todas as estruturas necessárias na identificação; $\mathrm{o} \mathrm{M}_{7}$, à família Laelapidae e ao gênero Androlaelaps sp. 2; $\mathrm{M}_{8}$, à família Ixodidae $\mathrm{e}$ ao gênero Ixodes sp. A identificação foi realizada, empregando-se a chave de identificação proposta por Furman (1972) e por Lane e Crosskey (1993). De acordo com a composição dos grupos $\mathbf{M}_{1}, \mathbf{M}_{2}, \mathrm{M}_{3}, \mathrm{M}_{4}, \mathrm{M}_{5}, \mathrm{M}_{6}, \mathrm{M}_{7}$ e $\mathrm{M}_{8}$ apresentados na Fig. 1, foi encontrado um total de 246 ectoparasitas do grupo $\mathrm{M}_{1}, 149$ do grupo $\mathrm{M}_{2}$, 54 do grupo $\mathrm{M}_{3}$, quatro do grupo $\mathrm{M}_{4}$, um do grupo $\mathrm{M}_{5}$, um do grupo $\mathrm{M}_{6}$, um do grupo $\mathrm{M}_{7}$, dois do grupo $\mathrm{M}_{8}$, totalizando 458 ectoparasitas analisados, sendo 454 Acari e quatro Siphonaptera.

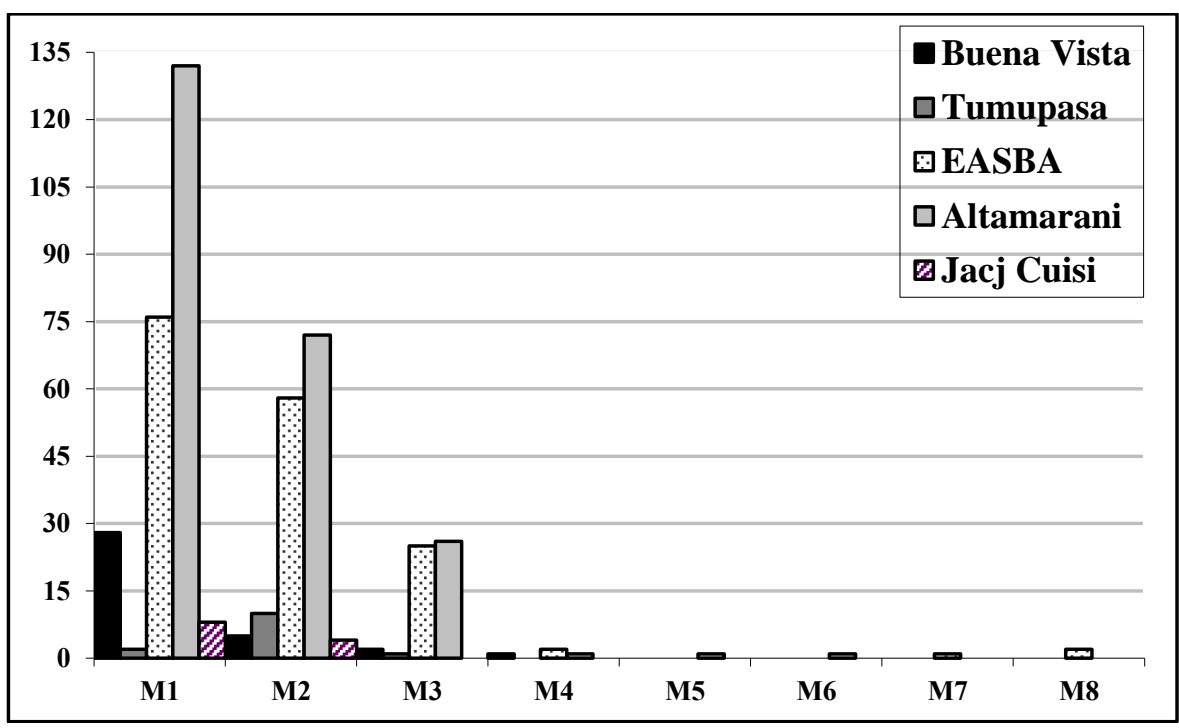

Figura 1. Quantidade de ectoparasitas coletados segundo características morfológicas de roedores do gênero Oligoryzomys microtis por região. 
Das três amostras de roedores analisadas de Buena Vista, dois roedores estavam parasitados com o grupo $\mathrm{M}_{1}$, um com o grupo $\mathrm{M}_{2}$, um com o grupo $\mathrm{M}_{3}$, dois com o grupo $\mathrm{M}_{4}$ e nenhum com os grupos $\mathbf{M}_{5}, \mathbf{M}_{6}, \mathbf{M}_{7}$ e $\mathbf{M}_{8}$. Das duas amostras de roedores analisadas de Tumupasa, um roedor estava parasitado com o grupo $\mathrm{M}_{1}$, dois com o grupo $\mathrm{M}_{2}$, um com o grupo $\mathrm{M}_{3}$ e nenhum com os grupos $\mathbf{M}_{4}, \mathrm{M}_{5}, \mathrm{M}_{6}, \mathrm{M}_{7}$ e $\mathrm{M}_{8}$. Das 15 amostras de roedores analisadas da EASBA, 14 roedores estavam parasitados com o grupo $\mathrm{M}_{1}, 13$ com o grupo $\mathrm{M}_{2}$, sete com o grupo $\mathrm{M}_{3}$, um com o grupo $\mathrm{M}_{4}$, nenhum com os grupos $\mathrm{M}_{5}$ e $\mathrm{M}_{6}$, um com os grupos $\mathbf{M}_{7}$ e $\mathbf{M}_{8}$. Das 15 amostras de roedores analisadas de Altamarani, 11 roedores estavam parasitados com o grupo $\mathrm{M}_{1}, 13$ com o grupo $\mathrm{M}_{2}$, 12 com o grupo $\mathrm{M}_{3}$, um com o grupo $\mathrm{M}_{4}$, um com o grupo $\mathrm{M}_{5}$, um com o grupo $\mathrm{M}_{6}$ e nenhum com os grupos $\mathbf{M}_{7}$ e $\mathbf{M}_{8}$. De uma amostra de roedor analisada de Jacj Cuisi, um roedor estava parasitado com os grupos $\mathrm{M}_{1}$ e $\mathrm{M}_{2}$ (Tab. 2).

Tabela 2. Quantidade de roedores da espécie Oligoryzomys microtis parasitados segundo características morfológicas de ectoparasitas coletados por região.

\begin{tabular}{lllllllll}
\hline \multicolumn{8}{c}{ Característica morfológica } \\
\hline Região & $\mathrm{M}_{1}$ & $\mathrm{M}_{2}$ & $\mathrm{M}_{3}$ & $\mathrm{M}_{4}$ & $\mathrm{M}_{5}$ & $\mathrm{M}_{6}$ & $\mathrm{M}_{7}$ & $\mathrm{M}_{8}$ \\
\hline Buena Vista & 02 & 01 & 01 & 02 & 0 & 0 & 0 & 0 \\
Tumupasa & 01 & 02 & 01 & 0 & 0 & 0 & 0 & 0 \\
EASBA & 14 & 13 & 07 & 01 & 0 & 0 & 01 & 01 \\
Altamarani & 11 & 13 & 12 & 01 & 01 & 01 & 0 & 0 \\
Jacj Cuisi & 01 & 01 & 0 & 0 & 0 & 0 & 0 & 0 \\
Total & 29 & 30 & 21 & 04 & 01 & 01 & 01 & 01 \\
\hline
\end{tabular}

No cálculo de abundância média dos roedores do gênero Oligoryzomys sp. com ectoparasitas, segundo as características morfológicas por região (Tab. 3), o grupo $M_{1}$ teve maior abundância em Buena Vista $(9,33)$, em Tumupasa (1), na EASBA $(5,07)$, em Altamarani $(8,8)$ e em Jacj Cuisi (8); no cálculo de prevalência da quantidade de roedores do gênero Oligoryzomys sp. parasitados por uma espécie específica de ectoparasitas, segundo as características morfológicas por região (Tab. 4), a região de Buena Vista teve o maior resultado nos grupos $\mathrm{M}_{1}$ e $\mathrm{M}_{4}$ (ambos 66,67\%); a de Tumupasa, no grupo $\mathrm{M}_{2}(100 \%)$; a da EASBA, no grupo $\mathrm{M}_{1}(93,33 \%)$; a de Altamarani, no grupo $\mathrm{M}_{2}(86,67 \%)$; a de Jacj Cuisi, nos grupos $\mathrm{M}_{1}$ e $\mathrm{M}_{2}(100 \%)$.

No cálculo de abundância média total de roedores do gênero Oligoryzomys sp. com ectoparasitas, segundo as características morfológicas (Tab. 3), o grupo $M_{1}$ teve a maior abundância média total $(6,83)$, seguido dos demais grupos. $\mathrm{O}$ grupo $\mathrm{M}_{2}$ apresentou a maior prevalência total $(83,33 \%)$ considerando o cálculo de prevalência total de roedores do gênero Oligoryzomys parasitados por uma espécie específica de ectoparasitas, segundo as características morfológicas,.

Tabela 3. Resultados da abundância média de roedores da espécie Oligoryzomys microtis com ectoparasitas, segundo as características morfológicas por região.

\begin{tabular}{lllllllll}
\hline \multicolumn{7}{c}{ Abundância média } \\
\hline Região & $\mathrm{M}_{1}$ & $\mathrm{M}_{2}$ & $\mathrm{M}_{3}$ & $\mathrm{M}_{4}$ & $\mathrm{M}_{5}$ & $\mathrm{M}_{6}$ & $\mathrm{M}_{7}$ & $\mathrm{M}_{8}$ \\
\hline Buena Vista & 9,33 & 1,67 & 0,67 & 0,33 & 0 & 0 & 0 & 0 \\
Tumupasa & 1 & 5 & 0,5 & 0 & 0 & 0 & 0 & 0 \\
EASBA & 5,067 & 3,87 & 1,67 & 0,13 & 0 & 0 & 0,07 & 0,13 \\
Altamarani & 8,8 & 4,8 & 1,73 & 0,07 & 0,07 & 0.07 & 0 & 0 \\
Jacj Cuisi & 8 & 4 & 0 & 0 & 0 & 0 & 0 & 0 \\
Abundância média total & 6,83 & 4,14 & 1,50 & 0,11 & 0,03 & 0,03 & 0,03 & 0,05 \\
\hline
\end{tabular}


Ocorrência de ectoparasitas...

Tabela 4. Resultados de prevalência da quantidade de roedores da espécie Oligoryzomys microtis parasitados por uma espécie específica de ectoparasitas, segundo as características morfológicas por região.

\begin{tabular}{|c|c|c|c|c|c|c|c|c|}
\hline \multicolumn{9}{|c|}{ Prevalência (\%) } \\
\hline Região & $\mathrm{M}_{1}$ & $\mathrm{M}_{2}$ & $\mathrm{M}_{3}$ & $\mathrm{M}_{4}$ & $\mathrm{M}_{5}$ & $\mathrm{M}_{6}$ & $\mathrm{M}_{7}$ & $\mathrm{M}_{8}$ \\
\hline Buena Vista & 66,67 & 33,33 & 33,33 & 66,67 & 0 & 0 & 0 & 0 \\
\hline Tumupasa & 50 & 100 & 50 & 0 & 0 & 0 & 0 & 0 \\
\hline EASBA & 93,33 & 86,67 & 46,67 & 6,67 & 0 & 0 & 6,67 & 6,667 \\
\hline Altamarani & 73,33 & 86,67 & 80 & 6,67 & 6,67 & 6,67 & 0 & 0 \\
\hline Jacj Cuisi & 100 & 100 & 0 & 0 & 0 & 0 & 0 & 0 \\
\hline Prevalência total (\%) & 80,56 & 83,33 & 58,33 & 11,11 & 2,78 & 2,78 & 2,78 & 2,78 \\
\hline
\end{tabular}

\section{DISCUSSÃO}

São consideradas como pequenos mamíferos todas as espécies que, na idade adulta, atingem o peso máximo de cinco quilogramas. Essa definição inclui as ordens Didelphimorphia, Insectivora, Rodentia, Lagomorpha, Carnívora e Chiroptera, sendo muito importante o papel ecológico nas cadeias alimentares nas comunidades, como predadores e presas dos pequenos mamíferos. Eles promovem impacto como herbívoros, tendo representatividade na biodiversidade, com a importante função como dispersores de sementes, hospedeiros de diversas espécies de endo e ectoparasitas, bem como o papel de reservatório de diferentes bioagentes (Oliveira et al., 2012). Os pequenos mamíferos (roedores, marsupiais e morcegos) constituem um grupo ecológico e economicamente importante, tanto por existirem em abundância e terem diversidade de espécies, quanto por serem encontrados como componentes fundamentais em quase todos os ecossistemas terrestres (Reis et al., 2008).

Considerando o número (221) de roedores capturados nas seis regiões-alvo deste trabalho de pesquisa, pode-se inferir que a abundância total de pequenos mamíferos não voadores encontrados pode estar relacionada, positivamente, à complexidade da vegetação, o que dificulta sobremaneira a prevenção de doenças veiculadas por eles (Oliveira et al., 2012). Além da importância numérica, os pequenos mamíferos não voadores exercem marcante influência na dinâmica das florestas neotropicais e são bons indicadores tanto de alterações locais do habitat como de alterações da paisagem. A influência na dinâmica da floresta se dá, principalmente, por meio da predação do banco de sementes e de plântulas, e da dispersão de sementes e fungos micorrízicos (Oliveira et al., 2012). Os animais tendem a ocupar, na maior parte do tempo, determinadas unidades da paisagem que lhes oferecem recursos necessários à sua sobrevivência, sendo os roedores um dos indicadores mais aptos para avaliação e monitoramento ambiental (Bonvicino et al., 2002; Oliveira et al., 2012).

Dos 458 ectoparasitas analisados, houve infestações simples e mistas, tendo maior presença da subclasse Acari por este trabalho ter sido realizado no período de outono-inverno, pois um maior número de Siphonaptera pode ser observado somente no período de primaveraverão (Barros-Battesti, 2008). Foram encontrados ectoparasitas do gênero Laelaps sp., Mysolaelaps sp., Gigantolaelaps sp., Polygenis sp., Androlaelaps sp. 1, Androlaelaps sp. 2 e Ixodes sp. Essas espécies também não diferiram das encontradas em outros estudos científicos (Barros-Battesti, 2008). Tal diversidade de ectoparasitas pode ocorrer, pois a disposição de cada espécie depende do tipo de flora da região, predispondo mais algumas espécies do que outras. Era esperado o que foi verificado neste estudo em relação às espécies de ectoparasitas da subclasse Acari por infestarem roedores; entretanto, elas podem, do mesmo modo, infestar outros hospedeiros (Barros-Battesti, 2008).

A abundância de ectoparasitas com seu hospedeiro depende da relação hospedeiroparasita, que pode estar relacionada à possibilidade de introdução do agente na área, ao grau de contaminação do ambiente, à resistência do agente, como também à exposição ao ectoparasita e à densidade e distribuição da população de hospedeiros, entre outros fatores. Nesse sentido, as regiões com maior abundância média de roedores com ectoparasitas, segundo as características morfológicas, foram Buena Vista (9,33), EASBA (5,07), Altamarani $(8,8)$ e Jacj Cuisi (8), com os gêneros Laelaps sp., e Tumupasa, com o gênero Mysolaelaps sp. (5). O 
gênero Laelaps sp. $(9,33)$ e o gênero Polygenis sp. $(0,33)$ tiveram maior abundância média na região de Buena Vista; o gênero Mysolaelaps sp., na região de Tumupasa (5); o gênero Gigantolaelaps sp. $(1,73)$, o gênero Androlaelaps sp. $(0,07)$ e a família Laelapidae do grupo $\mathrm{M}_{6}(0,07)$, na região de Altamarani; o gênero Androlaelaps sp. $(0,07)$ e o gênero Ixodes sp. $(0,13)$, em EASBA. Esses resultados de abundância média devem, mais provavelmente, estar relacionados ao bioma e ao clima de cada região, que, em cada uma delas, pode ou não favorecer cada um desses parasitas. Apesar de a captura dos roedores ter ocorrido no período de outono-inverno de todas as regiões estudadas, o clima era quente, o que propiciava ambiente favorável ao desenvolvimento dos ectoparasitas.

$\mathrm{Na}$ região de Jacj Cuisi, o único roedor Oligoryzomys microtis capturado estava parasitado com os gêneros Laelaps sp. e Mysolaelaps sp. (ambos $100 \%$ de prevalência). Dentro das áreas de captura, esses gêneros também tiveram a maior abundância média total e a maior prevalência total, tendo, ainda, o gênero Laelaps sp. (6,83) apresentado a maior abundância entre todos, e o gênero Mysolaelaps sp. $(83,33 \%)$ a maior prevalência. Tal fato pode se relacionar positivamente à disposição desses gêneros nessas regiões com a complexidade da vegetação. Como ressalta Oliveira et al. (2012), as características estruturais do ambiente são um dos fatores que influenciam a distribuição e a abundância de pequenos mamíferos. Entretanto, pelas limitações de qualquer método de colheita o que não é exceção daquele utilizado neste trabalho de pesquisa -, deve-se considerar a possibilidade da presença, no ambiente, de outros roedores, não capturados, que podem estar parasitados com outros gêneros de ectoparasitas.

A família Laelapidae foi aquela que apresentou a maior diversidade de gêneros. Na região da EASBA foi onde os roedores tinham maior quantidade de ectoparasitas, o que condiz com outro estudo em que os ácaros da família Laelapidae foram os que apresentaram maior diversidade de gêneros e hospedeiros (Reis et al., 2008). De acordo com Reis et al. (2008), em estudo com 36 roedores, foram encontrados os gêneros Androlaelaps sp., Laelaps sp. e Amblyomma sp. em Oligoryzomys microtis, não sendo encontrado Mysolaelaps sp. No presente trabalho, não foram encontrados ectoparasitas do gênero Amblyomma sp., porém foi encontrado Mysolaelaps sp. Barros-Battesti (2008) descreve a presença de Laelaps paulistanensis, Mysolaelaps parvispinosus e Gigantolaelaps wolffsohni em roedores Oligoryzomys nigripes, havendo concordância com os gêneros deste trabalho.

Do ponto de vista epidemiológico, os roedores são considerados um dos grupos de hospedeiros mais importantes quando se considera a possibilidade de eles se comportarem como reservatórios de microrganismos transmitidos, por exemplo, por pulgas, causadores de zoonoses, tais como a peste bubônica, o tifo murino e a tularemia (BarrosBattesti, 2008). Pulgas do gênero Polygenis apresentam nítida preferência por roedores, podendo, no entanto, proporcionar infestações secundárias em marsupiais e, acidentalmente, em outros grupos de hospedeiros (Reis et al., 2008). Essa preferência pelos roedores deve-se ao hábito nidícola, o que favorece o desenvolvimento do inseto tanto na fase parasitária quanto na fase não parasitária do seu ciclo biológico. Em estudo de Reis et al. (2008), as pulgas foram encontradas apenas em marsupiais, e não em roedores. Os autores enfatizam a não especificidade de hospedeiro entre as espécies da família Rhopalopsyllidae, embora a preferência alimentar por alguns hospedeiros tenha sido observada.

Estudos demonstram a presença de membros da família Rhopalopsyllidae na floresta atlântica, bioma que concentra o maior número de espécies de pulgas conhecidas, seguida da floresta de araucária (Linardi et al., 2005; Reis et al., 2008). Em outro estudo, as pulgas foram abundantes no bioma de floresta ombrófila mista (BarrosBattesti, 2008). Porém, na presente pesquisa, elas foram encontradas na floresta tropical amazônica, sendo, aparentemente, um novo relato sobre o ambiente em que pode viver o gênero Polygenis sp. da família Rhopalopsyllidae, fato importante por ser esse potencialmente transmissor da peste silvestre.

Áreas com grandes ofertas de alimentos refletem maior prevalência de roedores; isso pode ser observado por meio da alta prevalência de roedores capturados em área de cultivo $(86,11 \%)$, provavelmente devido à maior facilidade de busca por alimentos. Houve também maior prevalência de roedores na idade subadulta $(75 \%)$ do que adultos $(25 \%)$; isso pode ter ocorrido, pois, o inverno é a provável época de reprodução e os 
roedores subadultos estão à procura de seu par para reproduzir. $\mathrm{O}$ contínuo estudo sobre parasitismo por artrópodes em roedores é importante para a avaliação dos riscos à saúde pública e o monitoramento do impacto ambiental.

\section{CONCLUSÃO}

Considerando cinco regiões estudadas, Buena Vista, Tumupasa, Sadiri, Altamarani, EASBA e Jacj Cuisi - município de San Buenaventura de La Paz, Bolívia, foram identificados roedores do gênero Oligoryzomys microtis nas regiões de Buena Vista, Tumupasa, EASBA, Altamarani e Jacj Cuisi, encontrados em maior prevalência na área de cultivo. Entre todos os 458 ectoparasitas coletados, houve maior presença da subclasse Acari do que da Siphonaptera, sendo o gênero Laelaps sp. com maior abundância e Mysolaelaps com maior prevalência. $\mathrm{Na}$ floresta tropical amazônica, pela primeira vez, aparentemente, foi descrita a presença do gênero Polygenis sp., da família Rhopalopsyllidae.

\section{AGRADECIMENTOS}

Este trabalho foi realizado no âmbito do projeto INVESTIGACIÓN DE ENFERMEDADES ZOONÓTICAS EMERGENTES EN EL NORTE DE LA PAZ, financiado com fundos IDHUMSA, 2016-2018. Os autores agradecem aos guias de campo: Camilo Roble Gonzáles Racua, Ramiro Apana Beyuma, Julio Duval Chao Eladio Chao Lurisi, Gilberto Cartagena Chao, Raúl Navi Palomeque, Hernán Navi Palomeque, Vivian Anetty Macuapa, Rafael Canavari, Antolin Dibivay Cartagena, Rosario Barradas Cuqui y Wilson Boteano Navi, que ajudaram durante o trabalho de campo, para que fosse bem-sucedido.

\section{REFERÊNCIAS}

ANDERSON, S. Mammals of Bolivia, taxonomy and distribution. Bull. Am. Mus. Nat. Hist., n.231, p.1-652, 1997.

BARROS-BATTESTI, D.M. Biodiversidade de ectoparasitos de pequenos mamíferos e aves silvestres em biomas preservados e degradados no estado do Paraná. Relatório para o Instituto Ambiental do Paraná (IAP), 2008. p.54.
BONVICINO, C.R.; LINDBERGH, S.M.; MAROJA, L.S. Small non-flying mammals from conserved and altered areas of atlantic forest and cerrado: comments on their potential use for monitoring environment. Braz. J. Biol., vol.62, p.765-774, 2002.

BUSH, A.O.; LAFFERTY, K.D.; LOTZ, J.M.; SHOSTAK, A.W. Parasitology meets ecology on its own terms: Margolis et al. revisited. $J$. Parasitol., v.83, p.575-583, 1997.

CASTRO, V.; AZEVEDO, S.S.; GOTTI, T.B. et al. Fatores de risco para a leptospirose em fêmeas bovinas em idade reprodutiva no Estado de São Paulo. Arq. Bras. Med. Vet. Zootec., v.61, p.14381442, 2009.

ESTEVAM, G.; JOB, J.R.P.P. Animais exóticos domesticados com potencial zoonótico - Revisão de literatura. Rev. Soc. Bras. Clín. Méd., v.14, p.114-20, 2016.

FURMAN, D.P. Mites of the family Laelapidae in Venezuela (Acarina: Laelapidae). Brigham Young Univ. Sci. Bull. Biol. Ser., vol.17, p.2-57, 1972.

KÖRTING, K.S.; FLACH, J.; HONSCHA, G.; MARTINEZ, A.M.B. Hantavirose: patologia e registro no Brasil. Vittalle, v.20, p.39-50, 2008.

LANE, R.P.; CROSSKEY, R.W. Medical insects and arachnids. London: Chapman \& Hall. 1993. p.529-659.

LARESCHI, M.; LÓPEZ, M.I.S. Ectoparásitos (Phthiraptera y Acari) de roedores (Rodentia: Muridae: Sigmodontinae) en el delta bonaerense del río Paraná, Argentina. Rev. Soc. Entomol. Argent., v.59, p.17-19. 2000.

LINARDI, P.M.; CARDOSO, V.A.; BOTELHO, J.R. et al. Polygenis (Polygenis) platensis (Jordan \& Rothschild) (Siphonaptera: Rhopalopsyllidae, Rhopalopsyllinae), a new record in Brazil. Neotrop. Entomol., v.34, p.840, 2005.

MACHADO, L.F.; PARESQUE, R.; CHRISTOFF, A.U. Anatomia comparada e morfometria de Oligoryzomys nigripes e $O$. flavescens no Rio Grande do Sul, Brasil. Pap. Avulsos Zool., v.51, p.29-32, 2011.

OLIVEIRA, H.H.; GOMES, V.; AMORIM, M. et al. Diversidade de ixodida em roedores e marsupiais capturados no Parque Estadual da Pedra Branca, Rio de Janeiro, Brasil. Arq. Bras. Med. Vet. Zootec., v.66, p.1097-1104, 2014. 
OLIVEIRA, H.H.; SILVA, A.B., GOMES, V. et al. Roedores e marsupiais capturados no Parque Estadual da Pedra Branca, Rio de Janeiro: distribuição e relação com o ambiente. Revista UNIABEU, v.5, p.158-180, 2012.

REIS, F.S.; BARROS, M.C.; FRAGA, E.C. et al. Ectoparasitos de pequenos mamíferos silvestres de áreas adjacentes ao rio Itapecuru e área de preservação ambiental do Inhamum, estado do Maranhão, Brasil. Rev. Bras. Parasitol. Vet., v.17, p.69-74, 2008.
SILVA, J.C.R. Zoonoses e doenças emergentes transmitidas por animais silvestres. Associação Brasileira de Veterinários de Animais Selvagens/ABRAVAS, 2004. Disponível em: <www.abravas.org.br.>. Acessado em: 29 ago. 2017. 